\title{
Effect of Sowing Methodology on Diverse Hybrid Maize (Zea mays L.) Cultivars in Two Contrasting Environments
}

\author{
Pukhrajsingh*, Harmeetsingh Janeja and Sunita Rani
}

Department of Agronomy, School of Agriculture, Lovely Professional University, Phagwara, 144411, Punjab, India

*Corresponding author

\section{A B S T R A C T}

\section{Keywords}

Spring, Kharif, Hybrids, Flat/ridges

Article Info

Accepted:

15 June 2018

Available Online:

10 July 2018
A field experiment was conducted to identify best sowing methodology for different maize hybrids in two contrasting environments i.e. spring and kharif, 2017 at Lovely Professional University, Phagwara. In this experiment three factors, Season, varieties and flat/ridges sowing technique were studied as main, sub and sub-sub factors in split plot design with three replications and six popular hybrids. The data was recorded for Plant height, cob height $(\mathrm{cm})$, cob length $(\mathrm{cm})$, Cob breadth $(\mathrm{cm})$, Number of cobs/plot, Grain yield, cob weight/plot, Stubble weight, Harvest index\%. Relatively high magnitude of variation was observed for most of character from spring and kharif season. Among ridge/flat sowing methodology, it was found ridge sowing is better performed as compared to flat sowing in both seasons and most of hybrids (DKC9108 and Pioneer 1844). Similarly, DKC 9108 and Pioneer 1844 performed better in interactions of hybrids and sowing on different methods in term grain yield. Looking for both seasons, kharif season performed superior as compared spring season.

\section{Introduction}

Maize (Zea mays L.) is an important cereal crop and ranks third in production after wheat and rice in India. It is a plant is belongs to the family of grasses (Poaceae). It is cultivated globally one of most important cereal crop worldwide. It is called a queen of cereals. Maize is one of the world's leading crops cultivated over an area of about 139 mha with a production of about 600 mha of grain. Among the maize growing countries, USA has the largest area followed by Brazil, China, Mexico and India. In respect of production also USA stands first followed by China. In India, area, production and productivity of maize is $9.43 \mathrm{mha}, 24.35 \mathrm{mha}$ and $25.57 \mathrm{q} / \mathrm{ha}$ respectively. In Punjab, area, production and productivity of maize is $0.13 \mathrm{mha}, 0.50 \mathrm{mha}$ and $36.8 \mathrm{q} / \mathrm{ha}$ (Anonymous 2015 -16). Maize grain contains about 10 per cent protein, 4 per cent oil, 70 per cent carbohydrate, 2.3 per cent crude fiber, 10.4 per cent albuminoides, 1.4 per cent ash (Panchanathan et al., 2013). It can be helpful to crop body weight and milk quality in cattle due to higher nutrients value (chaudaryet al, 2016). Over $85 \%$ of maize produced in the country is consumed as 
human food. A variety of products such as starch, corn syrup, alcohol, acetic acid, glucose, lactic acid, paper, rayon, plastic, textile, corn oil, soaps, adhesive, dye, synthetic rubber and resin are produced form maize. Maize crop yields huge tonnage of green fodder, a good feed poultry, piggery and milch animals Maize crop based on the grain consumption different types of such as dent, flint, pop, pod, sweet, floury and waxy maize are suitable for specific usages. Among the different types of maize as sweet corn is most popular as green cobs. Maize grain consumption in boiled grains and vegetable purpose, it is also used for extracting sucrose as an industrial purpose. It is very profitable for rural farmers due to high cost of green cobs. It gives good return to the farmers and green stalk used as fodder.In Punjab maize grown in Rabi, Kharif and Spring seasons. Sowing of maize crop in many different methods like Dibbling, drilling and broadcasting in flat and ridges. Different planting methods including flat sowing, ridge sowing are employed for maize.

\section{Materials and Methods}

\section{Experimental detail}

This chapter includes the outlines of methodologies of experiment "Effect of sowing methodology on diverse hybrid maizecultivars in two contrasting environments." This was conducted at the research farms of Lovely Professional University, Phagwara, Punjab. During Spring and Kharif season of 2017-2018:The experiment was conducted at LPU field, School of Agriculture, Lovely Professional University, Phagwara, Punjab, during 20172018.: The experiment site is in sub-tropical region and has weather condition with hot summer and cool winters. The average temperature was $25-28^{\circ} \mathrm{c}$ at the crop growth stages which was optimum for crop growth and development. In experiment 6 treatments and three replications were used by split plot design. Six different hybrids were used viz namely Pioneer 1844, DKC 9108, DOW 2244, Rasi 4558, Kaveri 4210, Pioneer 1855. 8-10 $\mathrm{kg}$ seed rate per acre was used and sowing was done by flat and ridge sowing in two different spring and kharif seasons. Plant to plant $20 \mathrm{~cm}$ distance and row to row $60 \mathrm{~cm}$. the seed was sown about approximately depth of 4-6 cm. DAP was added in soil before the sowing of crop. Nitrogen was applied as basal dose as per treatment and remaining nitrogen in split doses at different critical stages of growth.

\section{Results and Discussion}

\section{Plant height}

Effect of between seasons, hybrids and sowing on different methods (flat/Ridges) on plant height $(\mathrm{cm})$

The comparison between seasons, hybrids and sowing on different methods (flat/Ridges) has been observed for plant height. The performance of hybrids in kharif season was higher as compared to spring season. During kharif season average height $(222.09 \mathrm{~cm})$ and spring season average height $(193.40 \mathrm{~cm})$. DKC $9108(228.5 \mathrm{~cm})$ is performed superior as compared to other in kharif season and DKC $9108(202.8 \mathrm{~cm})$ performed better in spring season. Among ridge/flat sowing methodology it was found that ridge sowing $(213.53 \mathrm{~cm})$ is better perform as compared to flat sowing $(202.95 \mathrm{~cm})$ in kharif season. At spring season Ridge sowing $(212.96 \mathrm{~cm})$ is better performed as compared to flat sowing (201.56). Across hybrid even though these were significantly different from each other (as per ANOVA analysis).

\section{Effect of season and hybrids on plant height}

The comparison between season and hybrids has been observed for plant height. Looking at both of season has been observed for plant 
height in which kharif season $\left(222.09 \mathrm{~cm}^{* *}\right)$ performed the better than spring season (193.40). DKC 9108 (228.5cm**) was performed superior as compared to other in kharif season and DKC $9108(202.8 \mathrm{~cm})$ was performed better in spring season. Across hybrids Even though these were significantly different from each other (as per ANOVA analysis at $5 \%$ and $1 \%$ level of significance).

\section{Effect of season and sowing on different methods (Flat/Ridges) on plant height}

The comparison between season and sowing on different methods (Flat/Ridges) has been observed plant height. The performance of hybrids in kharif season was higher as compared to spring season. kharif season average height $\left(222.09 \mathrm{~cm}^{* *}\right)$ and at spring season average height $\left(193.40 \mathrm{~cm}^{* *}\right)$. Among Ridge/Flat sowing methodology it was found that Ridge sowing $\left(213.53 \mathrm{~cm}^{* *}\right)$ is better performed as compared to flat sowing $(202.95 \mathrm{~cm})$ in kharif season. In spring season ridge sowing height $(212.96 \mathrm{~cm})$ and flat sowing height $(201.56 \mathrm{~cm})$. Across hybrids even though these were significantly different from each other (as per anova analysis as per $5 \%$ and $1 \%$ level of significance).

\section{Effect of hybrids and sowing on different methods (flat/ridges) on plant height $(\mathrm{cm})$}

The comparison between hybrids and sowing on different methods (flat/ridges). DKC 9108 performed superior than all the other hybrids has been observed for plant height in both season.

In comparison with DKC 9108(215.65cm) with other hybrids performed in followed by DOW 2244(211.77cm),Pioneer $1844(207.35 \mathrm{~cm})$, Pioneer $1855(206.78 \mathrm{~cm})$, Rasi 4558(203.6cm), kaveri 4210(201.32cm) has been observed for plant height. Among Ridge/flat sowing methodology it was found that Ridge sowing is performed superior as compared to flat sowing in both seasons. In kharif season average height at ridge sowing $(213.53 \mathrm{~cm})$ and flat sowing average height $(202.95 \mathrm{~cm})$ and in spring season average height at ridge sowing $(212.96 \mathrm{~cm})$ and flat sowing average height $(201.56 \mathrm{~cm})$. Across hybrids even though these were significantly different from each other (as per ANOVA analysis as per $5 \%$ and $1 \%$ level of significance).

\section{Cob length}

Effect of seasons, hybrids and sowing on different methods (flat/Ridges) on cob length $(\mathrm{cm})$

The comparison between seasons, hybrids and sowing on different methods (flat/Ridges) has been observed for cob length. The performance of hybrids in Kharif season is superior as compared to spring season. During kharif season average length $(16.04 \mathrm{~cm})$ and spring season average length $(15.75 \mathrm{~cm})$. Pioneer $1855(17.53 \mathrm{~cm})$ is performed superior as compared to other in Kharif season sowing in ridges and DKC $9108(16.73 \mathrm{~cm})$ better in spring season sowing in ridges. Among ridge/flat sowing methodology it was found that ridge sowing $(16.40 \mathrm{~cm})$ is better perform as compared to flat sowing $(16.06 \mathrm{~cm})$. Across hybrids even though these were non significantly different from each other (as per ANOVA analysis).

\section{Effect of season and hybrids on cob length (cm)}

The comparison between season and hybrids has been observed for cob length. Looking at both of season in length of cobs. During kharif season average length $(16.04 \mathrm{~cm})$ performed the better than spring season average length $(15.75 \mathrm{~cm})$. Pioneer $1844 \quad(16.85 \mathrm{~cm})$ was performed superior as compared to other in 
kharif season and DKC $9108(16.15 \mathrm{~cm})$ was performed better in spring season. Across hybrids even though these were non significantly different from each other (as per ANOVA analysis).

Effect of season and sowing on different methods (Flat/Ridges) on cob length $(\mathrm{cm})$

The comparison between season and sowing on different methods (Flat/Ridges) has been observed for cob length. kharif season is performed superior as compared to spring season. During kharif season average length $(16.04 \mathrm{~cm})$ and at spring season average length $(15.75 \mathrm{~cm})$. Among Ridge/Flat sowing methodology it was found that Ridge sowing is better performed as compared to flat sowing has been observed for cob length. Across hybrids even though these were non significantly different from each other (as per ANOVA analysis).

Effect of hybrids and sowing on different methods (flat/ridges) on cob length

The comparison between hybrids and sowing on different methods (flat/ridges). DOW 2244 performed superior than all the other hybrids has been observed for length of cobs. The comparison with DOW 2244 $(16.16 \mathrm{~cm})$ variety with other varieties performed in followed by Pioneer1844(16.16cm),DKC9108(16.15cm),Pi oneer $1855(15.85 \mathrm{~cm})$, Rassi $4558(15.67 \mathrm{~cm})$, Kaveri $4210(15.39 \mathrm{~cm})$ has been observed for length of cobs in $\mathrm{cm}$. Ridge sowing is performed superior as compared to flat sowing in both seasons.

During kharif season average length at ridge sowing $(16.40 \mathrm{~cm})$ and flat sowing average length $(15.69 \mathrm{~cm})$. During spring season average value at ridge sowing $(16.06 \mathrm{~cm})$ and flat sowing $(15.44 \mathrm{~cm})$. Across hybrids even though these were non significantly different from each other (as per ANOVA analysis).

\section{Number of cobs}

Effect of seasons, hybrids and sowing on different methods (flat/Ridges) on number of cobs

The comparison between seasons, hybrids and sowing on different methods (flat/Ridges) has been observed for number of cobs. The performance of hybrids in kharif season was higher as compared to spring season. During kharif season average cobs (33.44) and spring season average cobs (24.89). Pioneer 1855 (39) is performed superior as compared to other in kharif season sowing and Rasi 4558 (29.16) performed better in spring season. Among ridge/flat sowing methodology it was found that ridge sowing (30.80 cobs) is better perform as compared to flat sowing (25.22cobs). Across hybrids even though these were significantly different from each other (as per ANOVA analysis).

\section{Impact of season and hybrids on number of cobs}

The comparison between season and hybrids has been observed for number of cobs. Looking at both of season has been observed for number of cobs. The performance of hybrids in kharif season (33.44 cobs) is higher as compared to spring season (24.77 cobs). Pioneer $1855\left(39.5^{*}\right.$ cobs $)$ was performed superior as compared to other in kharif season and Rasi 4558 (29.16 cobs) was performed better in spring season. Across hybrids even though these were significantly different from each other (as per ANOVA analysis at 5\% level of significance).

\section{Effect of season and sowing on different methods (Flat/Ridges) on number of cobs}

The comparison between season and sowing on different methods (Flat/Ridges) has been observed for number of cobs. The 
performance of hybrids in kharif season is performed superior as compared to spring season. During kharif season average cobs (33.44 cobs) and spring season average cobs (24.77cobs). Among Ridge/Flat sowing methodology it was found that Ridge sowing (30.80 cobs) is better performed as compared to flat sowing (25.22cobs) in kharif season and spring season ridge sowing value (33.36 cobs) and flat sowing were $(27.05$ cobs). Across hybrids even though these were significantly different from each other (as per analysis 5\% level of significance).

\section{Effect of hybrids and sowing on different methods (flat/ridges) on number of cobs}

The comparison between hybrids and sowing on different methods (flat/ridges). Pioneer 1855 performed superior than all the other varieties has been observed for number of cobs in both season. A comparison with Pioneer 1855(32.45 cobs) variety other varieties performed in followed by Rasi 4558(30.04 cobs), DKC 9108(29.5cobs), Pioneer1844(27.91cobs), kaveri 4210 (27.87cobs), DOW 2244(26.87cobs) has been observed number of cobs. Among Ridge/flat sowing methodology it was found that Ridge sowing is performed superior as compared to flat sowing in both seasons. In kharif season average cobs at ridge sowing (33.36cobs) and flat sowing average cobs (27.05cobs). In spring season average cobs at ridge sowing (30.80cobs) and flat sowing average (25.22cobs). Across hybrids even though these were significantly different from each other (as per ANOVA analysis).

\section{Grain weight}

Effect of seasons, hybrids and flat/ridges on Grain yield (q/ha) in kharif

The comparison between seasons, varieties and sowing on different methods (flat/Ridges) has been studied for grain yield per hectare.
The performance of hybrids in Kharif season was higher as compared to spring season. During kharif season average grain yield $(40.27 \mathrm{q} / \mathrm{h})$ and spring season average grain yield (39.41 q/ha). DKC 9108 (67.15q/ha) is performed superior as compared to other in Kharif season and Pioneer 1855 (53.06q/ha) performed better in spring season. Among ridge / flat sowing methodology, it was found that ridge sowing $(47.33 \mathrm{q} / \mathrm{ha})$ is better as compared to flat sowing $(33.48 \mathrm{q} / \mathrm{ha})$ across hybrids even though these were nonsignificant different from each other (as per ANOVA analysis)

\section{Effect of seasons and hybrids on grain yield (q/ha)}

The comparison between season and hybrids has been of Grain weight. Looking at both of season has observed grain weight. The performance of hybrids in kharif season performed the better than spring season. DKC 9108 (49.51 q/ha) was performed superior as compared to other in kharif season and Pioneer 1844 (43.78 q/ha) was performed better in spring season. Across hybrids even though these were non significantly different from each other (as per ANOVA analysis).

\section{Effect of season and sowing methods (flat/ridges) on grain yield (q/ha)}

The comparison between season and sowing on different methods (Flat/Ridges). The performance of hybrids in kharif season was superior as compared to spring season. During kharif season average yield (40.27 q/ha) and spring season average yield (39.41 q/ha). Among Ridge sowing and Flat sowing methodology it was found that Ridge sowing is better performed as compared to flat sowing has been observed grain weight. Across hybrids even though these were non significantly different from each other (as per ANOVA analysis). 
Int.J.Curr.Microbiol.App.Sci (2018) 7(7): 2106-2120

Table.1 Effect of between seasons, hybrids and sowing on different methods (flat/Ridges) on plant height

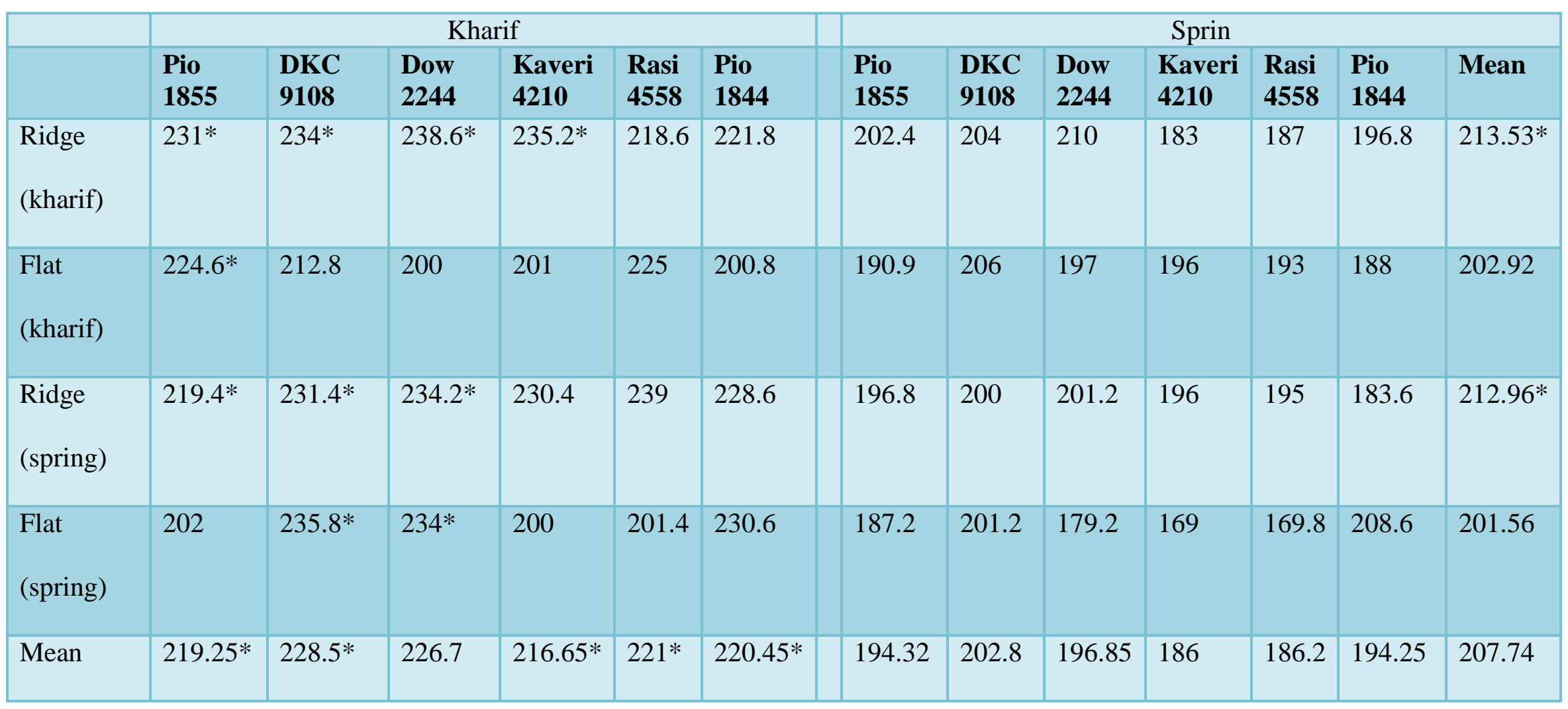


Effect of season and hybrids on plant height

\begin{tabular}{|l|l|l|l|l|l|l|l|}
\hline & Pio & DKC & Dow & Kaveri & Rasi & Pio & Mean \\
& 1855 & 9108 & 2244 & 4210 & 4558 & 1844 & \\
\hline Kharif & 219.25 & $228.5^{* *}$ & $226.7 * *$ & 216.65 & $221^{*}$ & $220.45^{*}$ & $222.09^{* *}$ \\
\hline Spring & $194.32^{*}$ & 202.8 & 196.85 & $186^{* *}$ & $186.2^{* *}$ & $194.25^{*}$ & 193.40 \\
\hline Mean & 206.78 & 215.65 & 211.77 & 201.32 & 203.6 & 207.35 & 207.74 \\
\hline
\end{tabular}

Effect of season and sowing on different methods (Flat/Ridges) on plant height

\begin{tabular}{|l|l|l|l|l|l|}
\hline & $\begin{array}{l}\text { Ridge } \\
\text { (kharif) }\end{array}$ & $\begin{array}{l}\text { Flat } \\
\text { (kharif) }\end{array}$ & $\begin{array}{l}\text { Ridge } \\
\text { (spring) }\end{array}$ & $\begin{array}{l}\text { Flat } \\
\text { (spring) }\end{array}$ & Mean \\
\hline Kharif & $229.86^{* *}$ & 210.7 & $230.5^{* *}$ & $217.3^{*}$ & $222.09^{* *}$ \\
\hline Spring & 197.2 & $195.15^{*}$ & $195.43^{*}$ & $185.83^{* *}$ & $193.40^{* *}$ \\
\hline Mean & $213.53^{* *}$ & 202.92 & 212.96 & 201.56 & 207.74 \\
\hline
\end{tabular}

Effect of hybrids and sowing on different methods (flat/ridges) on plant height

\begin{tabular}{|l|l|l|l|l|l|}
\hline & $\begin{array}{l}\text { Ridge } \\
\text { (kharif) }\end{array}$ & $\begin{array}{l}\text { Flat } \\
\text { (kharif) }\end{array}$ & $\begin{array}{l}\text { Ridge } \\
\text { (spring) }\end{array}$ & $\begin{array}{l}\text { Flat } \\
\text { (spring) }\end{array}$ & Mean \\
\hline $\begin{array}{l}\text { Pio } \\
\mathbf{1 8 5 5}\end{array}$ & $216.7^{*}$ & 207.75 & 208.1 & 194.6 & 206.78 \\
\hline $\begin{array}{l}\text { DKC } \\
\mathbf{9 1 0 8}\end{array}$ & $219^{*}$ & 209.4 & 215.7 & $218.5^{*}$ & $215.65^{*}$ \\
\hline $\begin{array}{l}\text { Dow } \\
\mathbf{2 2 4 4}\end{array}$ & $224.3^{*}$ & 198.5 & 217.7 & 206.6 & $211.77^{*}$ \\
\hline $\begin{array}{l}\text { Kaveri } \\
\mathbf{4 2 1 0}\end{array}$ & 209.1 & 198.5 & 213.2 & 184.5 & 201.32 \\
\hline $\begin{array}{l}\text { Rasi } \\
\mathbf{4 5 5 8}\end{array}$ & 202.8 & 209 & 217 & 185.6 & 203.6 \\
\hline $\begin{array}{l}\text { Pio } \\
\mathbf{1 8 4 4}\end{array}$ & 209.3 & 194.4 & 206.1 & $219.6^{*}$ & 207.35 \\
\hline Mean & $213.53^{*}$ & 202.92 & $212.96^{*}$ & 201.56 & 207.74 \\
\hline
\end{tabular}


Table.2 Effect of seasons, hybrids and sowing on different methods (flat/Ridges) on cob length

\begin{tabular}{|c|c|c|c|c|c|c|c|c|c|c|c|c|c|}
\hline & \multicolumn{6}{|c|}{ Kharif } & \multicolumn{6}{|c|}{ Spring } & \multirow[b]{2}{*}{ mean } \\
\hline & Pio 1855 & $\begin{array}{l}\text { DKC } \\
9108\end{array}$ & $\begin{array}{l}\text { Dow } \\
2244\end{array}$ & $\begin{array}{c}\text { Kaveri } \\
\mathbf{4 2 1 0}\end{array}$ & $\begin{array}{l}\text { Rasi } \\
4558\end{array}$ & $\begin{array}{c}\text { Pio } \\
1844\end{array}$ & $\begin{array}{c}\text { Pio } \\
1855\end{array}$ & $\begin{array}{l}\text { DKC } \\
9108\end{array}$ & $\begin{array}{l}\text { Dow } \\
2244\end{array}$ & $\begin{array}{c}\text { Kaveri } \\
\mathbf{4 2 1 0}\end{array}$ & $\begin{array}{l}\text { Rasi } \\
4558\end{array}$ & Pio 1844 & \\
\hline $\begin{array}{l}\text { Ridge } \\
\text { (spring) }\end{array}$ & 16.9 & 16.7 & 17.067 & 16.4 & 15.733 & 16.833 & 15.4 & 15.667 & 16.333 & 15.867 & 17.8 & 16.2 & 16.40833 \\
\hline $\begin{array}{c}\text { Flat } \\
\text { (spring) }\end{array}$ & 14.6 & 16.133 & 15.433 & 16.6 & 15.75 & 16.3 & 15.033 & 15.4 & 16.467 & 15.467 & 15.4 & 15.733 & 15.693 \\
\hline $\begin{array}{l}\text { Ridge } \\
\text { (Kharif) }\end{array}$ & 17.533 & 15.533 & 16.867 & 15.567 & 16.1 & 16.467 & 15.933 & 16.733 & 15.667 & 15.267 & 15.6 & 15.467 & 16.06117 \\
\hline $\begin{array}{c}\text { Flat } \\
\text { (Kharif) }\end{array}$ & 15.4 & 16.267 & 15.633 & 13.533 & 13.933 & 17.8 & 16 & 16.833 & 15.867 & 14.433 & 15.067 & 14.533 & 15.44158 \\
\hline mean & 16.10825 & 16.15825 & 16.25 & 15.525 & 15.379 & 16.85 & 15.5915 & 16.15825 & 16.0835 & 15.2585 & 15.96675 & 15.48325 & \\
\hline
\end{tabular}


Effect of season and hybrids on cob length

\begin{tabular}{|l|l|l|l|l|l|l|l|}
\hline & Pio & DKC & Dow & Kaveri & Rasi & Pio & Mean \\
& 1855 & 9108 & 2244 & 4210 & 4558 & 1844 & \\
\hline Kharif & 16.10 & 16.15 & 16.25 & 15.52 & 15.37 & 16.85 & 16.04 \\
\hline Spring & 15.59 & 16.15 & 16.08 & 15.25 & 15.96 & 15.48 & 15.75 \\
\hline Mean & 15.85 & 16.15 & 16.16 & 15.39 & 15.67 & 16.16 & 15.90 \\
\hline
\end{tabular}

Effect of season and sowing on different methods (Flat/Ridges) on cob length

\begin{tabular}{|l|l|l|l|l|l|}
\hline & $\begin{array}{l}\text { Ridge } \\
\text { (kharif) }\end{array}$ & $\begin{array}{l}\text { Flat } \\
\text { (kharif) }\end{array}$ & $\begin{array}{l}\text { Ridge } \\
\text { (spring) }\end{array}$ & $\begin{array}{l}\text { Flat } \\
\text { (spring) }\end{array}$ & Mean \\
\hline Kharif & 16.60 & 15.80 & 16.34 & 15.42 & 16.04 \\
\hline Spring & 16.21 & 15.58 & 15.77 & 15.45 & 15.75 \\
\hline Mean & 16.40 & 15.69 & 16.06 & 15.44 & \\
\hline
\end{tabular}

Effect of hybridsand sowing on different methods (flat/ridges) on cob length

\begin{tabular}{|l|l|l|l|l|l|}
\hline & $\begin{array}{l}\text { Ridge } \\
\text { (kharif) }\end{array}$ & $\begin{array}{l}\text { Flat } \\
\text { (kharif) }\end{array}$ & $\begin{array}{l}\text { Ridge } \\
\text { (spring) }\end{array}$ & $\begin{array}{l}\text { Flat } \\
\text { (spring) }\end{array}$ & Mean \\
\hline Pio 1855 & 16.15 & 14.81 & 16.73 & 15.7 & 15.85 \\
\hline DKC 9108 & 16.18 & 15.76 & 16.13 & 16.55 & 16.15 \\
\hline Dow 2244 & 16.7 & 15.95 & 16.26 & 15.75 & 16.16 \\
\hline Kaveri 4210 & 16.13 & 16.03 & 15.41 & 13.98 & 15.39 \\
\hline Rasi 4558 & 16.76 & 15.57 & 15.85 & 14.5 & 15.67 \\
\hline Pio 1844 & 16.51 & 16.01 & 15.96 & 16.16 & 16.16 \\
\hline Mean & 16.40 & 15.69 & 16.06 & 15.44 & \\
\hline
\end{tabular}


Int.J.Curr.Microbiol.App.Sci (2018) 7(7): 2106-2120

Table.3 Effect of seasons,hybrids and sowing on different methods (flat/Ridges) on number of cobs

\begin{tabular}{|c|c|c|c|c|c|c|c|c|c|c|c|c|c|}
\hline & \multicolumn{6}{|c|}{ Kharif } & \multicolumn{6}{|c|}{ Spring } & \multirow[b]{2}{*}{ Mean } \\
\hline & $\begin{array}{l}\text { Pio } \\
1855\end{array}$ & $\begin{array}{l}\text { DKC } \\
9108\end{array}$ & $\begin{array}{l}\text { Dow } \\
2244\end{array}$ & $\begin{array}{l}\text { Kaveri } \\
4210\end{array}$ & $\begin{array}{l}\text { Rasi } \\
4558\end{array}$ & $\begin{array}{l}\text { Pio } \\
1844\end{array}$ & $\begin{array}{l}\text { Pio } \\
1855\end{array}$ & $\begin{array}{l}\text { DKC } \\
9108\end{array}$ & $\begin{array}{l}\text { Dow } \\
2244\end{array}$ & $\begin{array}{l}\text { Kaveri } \\
4210\end{array}$ & $\begin{array}{l}\text { Rasi } \\
4558\end{array}$ & $\begin{array}{l}\text { Pio } \\
1844\end{array}$ & \\
\hline $\begin{array}{l}\text { Ridge } \\
\text { (kharif) }\end{array}$ & $39.66 *$ & $44.3 * 3$ & $35.33^{*}$ & $36^{*}$ & 26 & 29.33 & 28.66 & 21.66 & 25 & 26.66 & 24.66 & 32.33 & 30.80 \\
\hline $\begin{array}{l}\text { Ridge } \\
\text { (spring) }\end{array}$ & $46.33 *$ & $39.66^{*}$ & $41.66^{*}$ & $40.33^{*}$ & $36.66^{*}$ & 27.33 & 28.66 & 21.33 & 31.66 & 23 & 31.33 & 32.33 & 33.36 \\
\hline $\begin{array}{l}\text { Flat } \\
\text { (spring) }\end{array}$ & $36.33 *$ & 30.66 & 24 & 30 & 28.33 & 32.33 & 24 & 29.66 & 26 & 17.66 & 22.66 & 23 & 27.05 \\
\hline
\end{tabular}


Effect of season and hybrids on number of cobs

\begin{tabular}{|l|l|l|l|l|l|l|l|}
\hline & $\begin{array}{l}\text { Pio } \\
1855\end{array}$ & $\begin{array}{l}\text { DKC } \\
9108\end{array}$ & $\begin{array}{l}\text { Dow } \\
2244\end{array}$ & $\begin{array}{l}\text { Kaveri } \\
4210\end{array}$ & $\begin{array}{l}\text { Rasi } \\
4558\end{array}$ & Pio & Mean \\
\hline Kharif & $39.5^{*}$ & 36.58 & 30.33 & 33.83 & 30.91 & 29.5 & 33.44 \\
\hline Spring & 25.41 & 22.41 & 23.41 & 21.91 & 29.16 & 26.33 & 24.77 \\
\hline Mean & 32.45 & 29.5 & 26.87 & 27.87 & 30.04 & 27.91 & 29.11 \\
\hline
\end{tabular}

Effect of season and sowing on different methods (Flat/Ridges) on number of cobs

\begin{tabular}{|l|l|l|l|l|l|}
\hline & $\begin{array}{l}\text { Ridge } \\
\text { (kharif) }\end{array}$ & $\begin{array}{l}\text { Flat } \\
\text { (kharif) }\end{array}$ & $\begin{array}{l}\text { Ridge } \\
\text { (spring) }\end{array}$ & $\begin{array}{l}\text { Flat } \\
\text { (spring) }\end{array}$ & Mean \\
\hline Kharif & 35.11 & 29.72 & $38.66^{* *}$ & 30.27 & 33.44 \\
\hline Spring & 26.5 & 20.72 & 28.05 & 23.83 & 24.77 \\
\hline Mean & 30.80 & 25.22 & 33.36 & 27.05 & 29.11 \\
\hline
\end{tabular}

Effect of hybrids and sowing on different methods (flat/ridges) on number of cobs

\begin{tabular}{|c|c|c|c|c|c|}
\hline & $\begin{array}{l}\text { Ridge } \\
\text { (Kharif) }\end{array}$ & $\begin{array}{l}\text { Flat } \\
\text { (Kharif) }\end{array}$ & $\begin{array}{l}\text { Ridge } \\
\text { (spring) }\end{array}$ & $\begin{array}{l}\text { Flat } \\
\text { (spring) }\end{array}$ & Mean \\
\hline Pio 1855 & $37.5^{*}$ & 30.16 & $34.16^{*}$ & 28 & 32.45 \\
\hline $\begin{array}{l}\text { DKC } \\
9108\end{array}$ & 30.5 & 30.16 & 33 & 24.33 & 29.5 \\
\hline Dow 2244 & $36.66 *$ & 25 & 30.16 & $15.66 *$ & 26.87 \\
\hline $\begin{array}{l}\text { Kaveri } \\
4210\end{array}$ & 31.66 & 23.83 & 31.33 & 24.66 & 27.87 \\
\hline Rasi 4558 & $34 *$ & 25.5 & 25.33 & 35.33 & 30.04 \\
\hline Pio 1844 & 29.83 & 27.66 & 30.83 & 23.33 & 27.91 \\
\hline Mean & 33.36 & 27.05 & 30.80 & 25.22 & \\
\hline
\end{tabular}


Int.J.Curr.Microbiol.App.Sci (2018) 7(7): 2106-2120

Table.4 Effect of seasons, hybrids and flat/ridges on Grain yield

\begin{tabular}{|c|c|c|c|c|c|c|c|c|c|c|c|c|c|}
\hline & \multicolumn{6}{|c|}{ Kharif } & \multicolumn{6}{|c|}{ Spring } & \multirow[b]{2}{*}{ Mean } \\
\hline & $\begin{array}{l}\text { Pio } \\
1855\end{array}$ & $\begin{array}{l}\text { DKC } \\
9108\end{array}$ & $\begin{array}{l}\text { DOW } \\
2244\end{array}$ & $\begin{array}{l}\text { Kaveri } \\
4210\end{array}$ & $\begin{array}{l}\text { Rasi } \\
4558\end{array}$ & $\begin{array}{l}\text { Pio } \\
1844\end{array}$ & $\begin{array}{l}\text { Pio } \\
1855\end{array}$ & $\begin{array}{l}\text { DKC } \\
9108\end{array}$ & $\begin{array}{l}\text { DOW } \\
2244\end{array}$ & $\begin{array}{l}\text { Kaveri } \\
4210\end{array}$ & $\begin{array}{l}\text { Rasi } \\
4558\end{array}$ & $\begin{array}{l}\text { Pio } \\
1844\end{array}$ & \\
\hline Ridge(kharif) & 45.6 & 67.15 & 61.42 & 26.93 & 26.05 & 46.97 & 53.06 & 44.97 & 52.44 & 40.26 & 45.24 & 48 & 47.33 \\
\hline Flat(kharif) & 53.02 & 43.95 & 25.86 & 29.37 & 37.55 & 33.11 & 22.44 & 34.62 & 21.51 & 34.88 & 51.6 & 26.93 & 31.99 \\
\hline Ridge(spring) & 52.04 & 49.51 & 58.84 & 44.57 & 40.75 & 30.57 & 43.6 & 43.91 & 41.73 & 34.44 & 52.44 & 52.84 & 44.82 \\
\hline Flat(spring) & 33.46 & 37.42 & 30.53 & 32.48 & 25.73 & 33.64 & 34.17 & 43.15 & 31.95 & 22.13 & 23.73 & 45.73 & 33.48 \\
\hline Mean & 46.03 & 49.51 & 44.16 & 33.34 & 32.51 & 36.07 & 38.32 & 41.66 & 36.91 & 32.93 & 43.25 & 43.37 & 39.41 \\
\hline
\end{tabular}

Effect of seasons and hybrids on grain yield

\begin{tabular}{|l|l|l|l|l|l|l|l|}
\hline & Pio & DKC & DOW & Kaveri & Rasi & Pio & Mean \\
\hline kharif & 4855 & 9108 & 2244 & 4210 & 4558 & 1844 & \\
\hline Spring & 38.32 & 41.66 & 36.91 & 32.93 & 43.25 & 43.37 & 39.41 \\
\hline Mean & 42.17 & 45.58 & 40.53 & 33.13 & 37.88 & 39.72 & 39.84 \\
\hline
\end{tabular}


Effect of season and sowing methods (flat/ridges) on grain yield

\begin{tabular}{|l|l|l|l|l|l|}
\hline & Ridge(kharif) & Flat(kharif) & Ridge(spring) & Flat(spring) & Mean \\
\hline Kharif & 45.68 & 37.14 & 46.05 & 32.21 & 40.27 \\
\hline Spring & 47.33 & 32 & 44.83 & 33.48 & 39.41 \\
\hline Mean & 46.50 & 34.57 & 45.44 & 32.84 & \\
\hline
\end{tabular}

Effect of hybrids and sowing on different methods (flat/ridges) on grain yield (q/ha)

\begin{tabular}{|l|l|l|l|l|l|}
\hline & $\begin{array}{l}\text { Ridge } \\
\text { (kharif) }\end{array}$ & $\begin{array}{l}\text { Flat } \\
\text { (kharif) }\end{array}$ & $\begin{array}{l}\text { Ridge } \\
\text { (spring) }\end{array}$ & $\begin{array}{l}\text { Flat } \\
\text { (spring) }\end{array}$ & Mean \\
\hline Pio 1855 & 49.33 & 37.73 & 47.82 & 33.82 & 42.17 \\
\hline DKC 9108 & 56.97 & 39.28 & 46.71 & 40.28 & 45.58 \\
\hline DOW 2244 & 56.93 & 23.68 & 50.28 & 31.24 & 40.53 \\
\hline Kaveri 4210 & 33.6 & 32.13 & 39.51 & 27.31 & 33.13 \\
\hline Rasi 4558 & 35.62 & 44.57 & 46.6 & 24.73 & 37.88 \\
\hline Pio 1844 & 47.48 & 30.02 & 41.71 & 39.68 & 39.72 \\
\hline Mean & 46.50 & 34.57 & 45.44 & 32.84 & \\
\hline
\end{tabular}

Effect of hybrids and sowing on different methods (flat/ridges) on grain yield ( $q / h a)$

The comparison between hybrids and sowing on different methods (flat/ridges). DKC 9108 performed superior than all the other has been observed for grain weight in kharif season. The comparison with DKC 9108(45.58q/ha) with other hybrids performed followed by Pioneer1855 (42.17q/ha), DOW2244 (40.53q/ha), pioneer (39.72q/ha), Rasi4558 (37.38q/ha), kaveri4210 (33.13q/ha) has been observed grain weight quintal per hectare. Among Ridge/flat sowing methodology it was found that Ridge sowing is performed superior as compared to flat sowing in both seasons. During kharif season average yield at ridge sowing $(46.50 \mathrm{q} / \mathrm{ha})$ and flat sowing average yield $(34.57 \mathrm{q} / \mathrm{ha})$. During spring season average yield at ridge sowing $(45.44 \mathrm{q} / \mathrm{ha})$ and flat sowing average yield $(32.84 \mathrm{q} / \mathrm{ha})$. Across hybrids even though these were non significantly different from each other (as per ANOVA analysis).

In conclusion, ridge planting method exhibit better grain yield as well as grain contributing characters namely cob weight, number of cobs, cob length, cob height, stubble weight and harvest index in both seasons (kharif and spring). DKC 9108 performed better as compared to other hybrids in both kharif seasons whereas Pioneer 1844 was performed superior in spring season.

Kharif season shows better results as compared to spring season for performance of all hybrids. The ridge sowing methodology 
was more efficient as compared to flat sowing in our findings. In ridge sowing, agronomic practices were conducted easily as compared to flat sowing. In ridge sowing, fertilizers and pesticide application was easier and reduces losses in addition to relatively better control of weeds. The irrigation management was easier and more. So DKC 9108 hybrid, sown in kharif season on ridges has been recommended on basis of our results. In DKC 9108 hybrid highest benefit cost ratio (1.70) as compared to other hybrids

\section{References}

Afzal, M. A. U. H., Ahmad, a. U. H., and zamir, s. I. (2013). Performance of multicut forage sorghum under various sowing methods and nitrogen application rates. The journal of animal and plant sciences, 23(1), 232239.

Akmal, M., Rehman, H., Farhatullah, M. A., and Akbar, H. (2010). Response of maize varieties to nitrogen application for leaf area profile, crop growth, yield and yield components. Pak. J. Bot, 42(3), 1941-1947.

Ali, M. K., Alam, M. N., Barkotulla, M. A. B., Khandaker, S. M. A. T., and Simon, P. W. (2014) Effect of Earthing up and Level of Irrigation on Yield and Quality Seed Production of Onion. Progressive Agriculture, 18(2), 81-91.

Amin, M. T., Anjum, L., Alazba, A. A., and Rizwan, M. (2015). Effect of the irrigation frequency and quality on yield, growth and water productivity of maize crops. Quality Assurance and Safety of Crops and Foods, 7(5), 721730.

Bakht, J., Siddique, M. F., Shafi, M., Akbar, H., Tariq, M., Khan, N., ...\& Yousef, M. (2007). Effect of planting methods and nitrogen levels on the yield and yield components of maize. Sarhad Journal of Agriculture, 23(3), 553.

Belay, Y. (1998). Effect of tied ridges on grain yield response of Maize (Zea mays L.) to application of crop residue and residual $\mathrm{N}$ and $\mathrm{P}$ oil two soil types at Alemaya, Ethiopia. South African Journal of Plant and Soil, 15(4), 123129.

Brar, H. S., Khehra, A. S., Sharma, R. K., and Dhillon, B. S. (1988). Effect of sowing dates, method of sowing and row spacing on maize varieties in winter. Indian J Eco, 15(1), 62-65.

Chandrasekaran, B., Annadurai, K., and Somasundaram, E. (2010). A textbook of agronomy. New Age International Limited.

Ehsanullah, U., Anjum, S. A., Ehsan, F., Khan, I., and Ghaffar, A. (2015) Tillage practices and sowing methods affect yield and related attributes of maize. Asian J. Agric. Biol, 3(1): 8-14.

Gul, S., Khan, M. H., Khanday, B. A. \&Nabi, S. (2015). Effect of sowing methods and NPK levels on growth and yield of rainfed maize (Zea mays L.). Scientific, 2015.

Gul, S., khan, M. H., khanday, B. A., and Nabi, S. (2015). Effect of sowing methods and npk levels on growth and yield of rainfed maize (Zea mays 1.). Scientifica, 2015.

Gwenzi, W., Taru, M., Mutema, Z., Gotosa, J., and Mushiri, S. M. (2008). Tillage system and genotype effects on rainfed maize (Zea mays L.) productivity in semi-arid Zimbabwe. African Journal of Agricultural Research 3(2) 101-110.

Hussain, N., Jamal, M., Hassan, G., and Yaqoob, M. (1999). Effect of methods of sowing and cultural practices on maize. Sarhad Journal of Agriculture (Pakistan).

Hussain, N., Jamal, M., Hassan, G., and 
Yaqoob, M. (1999). Effect of methods of sowing and cultural practices on maize. Sarhad Journal of Agriculture (Pakistan).

Jassal, R. K. (2013). Effect of planting methods and nitrogen levels on the seed production of fodder maize (Doctoral dissertation, Punjab Agricultural University, Ludhiana).

Kaur, A. (2016). Effect of different planting methods and nitrogen levels on the growth, yield and quality of kharif maize (Zea mays L.) (Doctoral dissertation, Punjab Agricultural University, Ludhiana)

Kaur, j., and bons, h. K. (2017). Mulching: a viable option to increase productivity of field and fruit crops. Journal of applied and natural science, 9(2), 974-982.

Kaur, M. (2011). Growth, quality and water productivity of August sown maize as affected by planting method, mulch and irrigation regimes (Doctoral dissertation, Punjab Agricultural University, Ludhiana).

Kaur, T. (2002). Studies on irrigation requirement in relation to method of planting of maize (Zea mays L.) (Doctoral dissertation, M. Sc.
Thesis, Punjab Agricultural University, Ludhiana, India).

Kaur, T., \&Mahey, R. K. (2006). Effect of Planting Methods and Irrigation Levels on Water Use of Maize (Zea mays, L.). Green Technologies for Sustainable Agriculture, 286.

Khan, M. B., Rafiq, R., Hussain, M., Farooq, M., \&Jabran, K. (2012) Ridge sowing improves root system, phosphorus uptake, growth and yield of Maize (Zea mays L.) Hybrids. Measurements, 22, 309-317.

Khehra, A. S., Dhillon, B. S., Saxena, V. K., Malhi, N. S., Kapoor, W. R., Pal, S. S., and Malhotra, V. V. (1981). Studies on winter maize in Punjab. In Proc. Seventh Rabi Maize Workshop. All India Coordinated Maize Improvt. Proj., New Delhi, India (pp. 72-80).

Kumar, V., Sharma, V., Sharma, S. C., and Singh, S. (2017). Performance of high yielding drought tolerant varieties of maize and in situ moisture conservation techniques in Kandi region of Punjab, India. Journal of Applied and Natural Science,9(1), 316-32.

\section{How to cite this article:}

Pukhrajsingh, Harmeetsingh Janeja and Sunita Rani. 2018. Effect of Sowing Methodology on Diverse Hybrid Maize (Zea mays L.) Cultivars in Two Contrasting Environments. Int.J.Curr.Microbiol.App.Sci. 7(07): 2106-2120. doi: https://doi.org/10.20546/ijcmas.2018.707.248 